\title{
Cost effectiveness of robotic mitral valve surgery
}

\author{
Emmanuel Moss ${ }^{1}$, Michael E. Halkos ${ }^{2}$ \\ ${ }^{1}$ Division of Cardiac Surgery, Jewish General Hospital, Montreal, Quebec, Canada; ${ }^{2}$ Cardiothoracic Surgery, Emory University School of Medicine, \\ Atlanta, Georgia, USA \\ Correspondence to: Emmanuel Moss, MD, MSc, FRCSC. Assistant Professor of Surgery, McGill University; Division of Cardiac Surgery, Jewish \\ General Hospital, Suite A-520, 3755 Cote Ste Catherine Ave, Montreal, Quebec H3T 1E2, Canada. Email: emmanuel.moss@mcgill.ca; Michael \\ E. Halkos, MD, MSc. Associate Professor, Cardiothoracic Surgery, Emory University School of Medicine, Medial Office Tower, 6th Floor, 550 \\ Peachtree Street NE, Atlanta, Georgia 30308, USA. Email: mhalkos@emory.edu.
}

\begin{abstract}
Significant technological advances have led to an impressive evolution in mitral valve surgery over the last two decades, allowing surgeons to safely perform less invasive operations through the right chest. Most new technology comes with an increased upfront cost that must be measured against postoperative savings and other advantages such as decreased perioperative complications, faster recovery, and earlier return to preoperative level of functioning. The Da Vinci robot is an example of such a technology, combining the significant benefits of minimally invasive surgery with a "gold standard" valve repair. Although some have reported that robotic surgery is associated with increased overall costs, there is literature suggesting that efficient perioperative care and shorter lengths of stay can offset the increased capital and intraoperative expenses. While data on current cost is important to consider, one must also take into account future potential value resulting from technological advancement when evaluating cost-effectiveness. Future refinements that will facilitate more effective surgery, coupled with declining cost of technology will further increase the value of robotic surgery compared to traditional approaches.
\end{abstract}

Keywords: Robotic; mitral valve; cost effectiveness

Submitted Oct 14, 2016. Accepted for publication Oct 30, 2016.

doi: 10.21037/acs.2017.01.03

View this article at: http://dx.doi.org/10.21037/acs.2017.01.03

There has been tremendous evolution and innovation in cardiac surgery over the last half century. In the early years of the specialty, innovation focused on decreasing mortality and expanding the pathologies that surgeons could address during heart operations. In the current era, with operative mortality for routine procedures exceedingly low, the focus has shifted to decreasing perioperative complications, improving perioperative quality of life (QOL), and maximizing long-term outcomes. Additionally, the rapid evolution of transcatheter technologies has meant that surgeons are not only measured against historical and contemporary surgical results. Patients and cardiologists are tempted by the allure of less invasive operations with shorter recovery, at times showing less concern for whether a procedure is equally effective in the short- or long-term. As a result, the onus has fallen on surgeons to evolve away from sternotomies and offer equally effective operations through less invasive approaches.

Mitral valve surgery (MVR) is one area that has seen some of the most impressive progress over the last two decades. With the advent of new technology, including peripheral cannulation systems, specially designed instruments, and robotic-assistance, complex valve repair and replacement can now be performed through small incisions in the right chest without disturbing the skeleton. Minimally traumatic surgical approaches offer patients gold standard results with fewer complications and a faster recovery, ensuring that despite the growth of transcatheter technologies, patients and cardiologists will not have to make the choice of trading long-term efficacy for shortterm gains. Although minimally invasive surgery brings with it many potential advantages, $21^{\text {st }}$ century surgical innovation is often associated with increased up-front costs. In the current economic healthcare climate, the perceived 
increase in costs associated with robotic-assisted surgery has led to questions about its sustainability and overall value. When compared to traditional sternotomy approaches, the cost disparity, if indeed one does exist, must be evaluated against the potential benefit to the patient. In the case of robotic mitral valve surgery, this must go beyond traditional measures of perioperative costs and evaluate not only QOL but consider the less tangible benefit of offering patients a minimally invasive "gold standard" intervention that is an attractive alternative to as yet inferior transcatheter technology.

The costs surrounding robotic surgery can be deconstructed into four broad categories: capital costs, operative costs, postoperative costs, and post-hospital costs. In non-single payer healthcare systems such as the United States, costs are often discussed in economical terms and referred to as direct or indirect. Direct costs are those which generate revenue, and indirect costs are overheads and do not generate revenue. Costs are then broken down into variable or fixed. Variable costs are those that occur or fluctuate because of that specific usage, while fixed costs are those that remain stable regardless of usage. Disposables such as robotic instruments and cannulae are examples of variable costs. Indirect cost items must be allocated over the direct cost items that do produce revenue. Included within the indirect costs is depreciation expense for all associated capital outlays, including those from the da Vinci robot utilized during robotic mitral valve surgery. The cost of purchasing the da Vinci robot can vary between $\$ 1.5$ and $\$ 2.5$ million, depending on specific contracts and the purchase of accessories. This leads to an inevitable increase in fixed capital costs with robotic surgery compared to other approaches. However, when analyzing the impact on a "per user" basis, the burden can be minimized by ensuring maximum usage of the robot, either by the cardiac surgery team or, as is more often the case, by other surgical specialties as well. Thus, more often than not, the capital costs associated with purchasing a robotic system are usually distributed to multiple services. Operative costs with robotic surgery will also be increased compared to nonrobotic approaches, particularly early in a team's learning curve. Once the learning curve has been negotiated, the need for specialized disposable equipment will maintain the increased intraoperative costs. In light of fixed increased capital and intraoperative costs, significant savings and/ or relative improvement in QOL must occur in the postoperative period in order for robotic-assisted surgery to be cost effective. Postoperative savings can be accrued by two primary mechanisms, decreased utilization of resources for a comparable uncomplicated postoperative course, or a decrease in complication rates compared to the traditional approach. Comparative studies have suggested that roboticassisted surgery does indeed decrease resource utilization in the postoperative period, and is associated with a decrease in some perioperative complications (1). These include reduced need for blood transfusion, shorter ventilator and intensive care times, elimination of sternal complications, and shorter hospital stays.

One of the earliest reports evaluating cost of robotic surgery was by the group from Columbia University in 2005. They reviewed a group of 40 patients who underwent either atrial septal defect or mitral valve repair using a robotic $(n=20)$ or sternotomy $(n=20)$ approach (2). When excluding capital costs, the total procedural cost was equivalent between the two approaches, with a minimal intraoperative increase in cost being offset by postoperative savings. However, when the analysis included amortized capital costs of the da Vinci system, robotic-assisted surgery was associated with an increased cost of $\$ 3,444$ per patient. An Australian study reached a similar conclusion, showing no increase in perioperative costs when excluding the initial cost of purchasing the robot (3). Suri and colleagues (4) evaluated whether affordability of robotic-assisted MVR was improved as a result of a cardiac surgery-wide surgical process improvement program. Interestingly, while robotic surgery at their institution was associated with increased cost prior to implementation of the changes $(\$ 34,920$ vs. $\$ 32,650, \mathrm{P}<0.001)$, the cost difference disappeared after implementing protocols that improved efficiency in operating room management and standardized postoperative care $(\$ 30,606$ vs. $\$ 31,310, \mathrm{P}=0.876)$. Savings in the robotic group were primarily related to earlier extubation, and shorter ICU and hospital lengths of stay. Unlike the earlier reports, this analysis included the amortized cost of the robot. A systematic review published in 2015 summarized the results of the above studies and concluded that total hospital costs of robotic MVR are slightly higher than conventional sternotomy, and significantly higher if amortization is taken into account (5).

Hassan and colleagues published a unique study comparing costs of non-robotic minimally invasive MVR (mini-MVR) with robotic-assisted MVR (6). The study reported the procedural costs in relation to conventional surgery and found that robotic surgery was associated with an increase of \$2,064 per case, while mini-MVR increased costs by only $\$ 271$ per case. Although this as an interesting 
comparison, the study has significant limitations, most notably the use of datasets from multiple studies to form their conclusion, without a single study directly comparing the two techniques. The authors also rely on estimations for postoperative length of stay, which is an important driver of postoperative cost. A review of our institution's data found fiscal outcomes to be similar when comparing sternotomy and robotic approaches (7). The increased procedural costs were offset by lower postoperative resource utilization resulting from decreased transfusion requirements, shorter intubation times, and shorter ICU and hospital lengths of stay. The cost of transfusing a single unit of blood has been estimated at \$522-\$1,183 USD (8). In the largest reported robotic-assisted mitral valve series (9), the rate of perioperative blood product transfusions was $22 \%$, significantly lower than most reported in the literature following sternotomy.

While there is no data comparing readmission rates with robotic surgery compared to sternotomy, this information can be extrapolated from data comparing mini-thoracotomy and sternotomy mitral valve surgery. Grossi and colleagues (10) found that readmissions were significantly reduced at both 30 and 90 days compared to sternotomy. This can amount to significant savings in single-payer socialized medical system. It is also becoming of increasing importance in a system such as the United States, where new legislation may lead to penalties for readmission and bundled payments may eliminate reimbursements for readmissions and/or prolonged postoperative care.

The most complete evaluation of cost surrounding robotic MVR came in a recent publication by Mihaljevic and colleagues (11). They compared robotic MVR with three non-robotic approaches: full sternotomy, partial sternotomy, and minithoracotomy. In addition to operative and postoperative costs, the authors included data on patient's return to work following surgery. By stratifying patients according to income and using the United States Internal Revenue Service database, the cost value associated with an earlier return to work was estimated. The authors found that although robotic surgery was associated with higher procedural costs, postoperative costs were significantly lower and patients returned to work earlier (35 days versus for 49,56 , and 42 days for sternotomy, partial sternotomy, minithoracotomy, respectively). The total cost of care while in hospital, including total hospital direct costs plus amortized capital investment and maintenance costs of the robot, were $26.8 \%$ higher with robotic surgery than sternotomy. The cost was $32.1 \%$ higher than partial sternotomy and $20.7 \%$ higher than anterolateral thoracotomy. Conversely, patients returned to work sooner after robotic surgery compared to alternative approaches, on average $28.6 \%$ sooner than sternotomy and $37.5 \%$ sooner than partial sternotomy. Income related savings partially offset the increased hospital costs of robotic surgery, resulting in a net increased cost ranging from $14.8 \%$ to $15.7 \%$ with robotic surgery compared to alternative approaches. Although it is helpful to have a global understanding of all costs related to robotic surgery, figures including amortized costs are highly dependent on usage of the robot and will vary greatly between institutions. The amortized costs estimated in this study are based on use by a single service, cardiac surgery. The authors accurately comment that in such a context a minimal number of annual cases [55-100] must be performed in order for the procedure to remain cost effective. In the majority of institutions; however, capital costs of the robot are spread across multiple surgical specialties and thus a smaller volume robotic cardiac surgery program can be economically viable. Regardless of how the capital cost is rationalized, it is unfair from an economic standpoint to consider this cost in a cost effectiveness analysis. The purchase of a robot is a "sunk cost", meaning a cost that has already been incurred and cannot be recovered. This can be compared to the cost of building a hybrid operating room to facilitate transcatheter aortic valve replacement (TAVR). The cost of a hybrid operating room is not incorporated into analyses when comparing TAVR to traditional aortic valve replacement, so why should robotic MVR be held to a different standard?

Overall, it appears that robotic mitral valve procedures tend to cost slightly more than open surgery, particularly if capital costs are considered. However, by focusing on streamlining care and taking into account "post-hospital" benefits, the cost difference can be minimized or abolished. The few studies that have made QOL comparisons have favored robotic surgery. While it is difficult to quantify this benefit in relation to procedural cost, the importance cannot be ignored (12). The current literature also has limited value in assessing the impact of rare complications of sternotomy surgery, such as deep sternal wound infection. The incidence is reportedly in the range of $0.27-1.30 \%$ following MVR and is associated with a cost of over US $\$ 33,000$ per patient affected $(13,14)$.

The Canadian Agency for Drugs and Technologies in Health published a report in 2011 providing an economic analysis of robotic-assisted surgery across multiple 
specialties (15). The report estimated cardiac surgery to be the least costly amongst all specialties, with a net program cost of $\$ 0.9$ million over 7 years. The favorable program costs were attributed primarily to improved hospital stay savings relative to other specialties. According to the report, if the life of a robot is extended from 7 to 10 years, net program costs would be even more favorable.

Although robotic MVR may be associated with increased cost at this time, any evaluation of a relatively new technology must assess not only its current value but also future potential that will inevitably result from continued technological advancement. In addition to declining cost of the technology, future refinements will facilitate more effective surgery and further increase the value compared to traditional approaches. Furthermore, as individual surgeons gain more experience, the technology will be applied to a broader range of patients with more complex valve disease and comorbid conditions. The current literature has shown that robotic surgery leads to postoperative savings, despite the fact that these studies have included a relatively young and healthy patient population. Murphy and colleagues (9) have shown that robotic-assisted MVR can be safely applied to a higher risk population, including those with advanced age, previous sternotomy, significant pulmonary hypertension, and requiring concomitant procedures. The greatest clinical and economic benefit with robotic surgery will likely be seen when the technology is applied more broadly to these "sicker" patients. One further example of potential societal savings is a lower requirement for transfer to a postoperative rehabilitation or convalescence, with a rate of only $2.9 \%$ in the above-referenced report.

The available literature has clearly shown that the costs associated with robotic-assisted MVR are in no way prohibitive. In our view, the potentially increased costs relative to traditional approaches are easily offset by the many advantages of the evolving technology. While many surgeons that have not embraced or had experience with robotic surgery argue that robotic-assistance does not enhance their ability to successfully perform a mitral valve repair, the advantages with regard to visualization and instrumentation of the mitral valve and subvalvular apparatus are clear. Regardless of technical and clinical advantages offered by the robot, patients are weary of undergoing a median sternotomy and, if not offered a less invasive surgical alternative, may be content with an inferior clinical result offered by transcatheter devices. A minimally invasive robotic approach gives patients an alternative to sternotomy without clinical compromise. Currently available clinical and cost data supports continued use of robotic technology for mitral valve surgery. Given the present cost-conscious healthcare climate, the appraisal of economics of robotic surgery will only intensify and, as adoption broadens and more surgeons become facile with the technique, the balance will likely continue to move in favor of this impressive technology.

\section{Acknowledgements}

None.

\section{Footnote}

Conflicts of Interest: The authors have no conflicts of interest to declare.

\section{References}

1. Mihaljevic T, Jarrett CM, Gillinov AM, et al. Robotic repair of posterior mitral valve prolapse versus conventional approaches: potential realized. J Thorac Cardiovasc Surg 2011;141:72-80.e1-4.

2. Morgan JA, Thornton BA, Peacock JC, et al. Does robotic technology make minimally invasive cardiac surgery too expensive? A hospital cost analysis of robotic and conventional techniques. J Card Surg 2005;20:246-51.

3. Kam JK, Cooray SD, Kam JK, et al. A cost-analysis study of robotic versus conventional mitral valve repair. Heart Lung Circ 2010;19:413-8.

4. Suri RM, Thompson JE, Burkhart HM, et al. Improving affordability through innovation in the surgical treatment of mitral valve disease. Mayo Clin Proc 2013;88:1075-84.

5. Canale LS, Colafranceschi AS. Is robotic mitral valve surgery more expensive than its conventional counterpart? Interact Cardiovasc Thorac Surg 2015;20:844-7.

6. Hassan M, Miao Y, Lincoln J, et al. Cost-benefit analysis of robotic versus nonrobotic minimally invasive mitral valve surgery. Innovations (Phila) 2015;10:90-5.

7. Moss E, Murphy D, Harskamp R, et al. Impact of Robotic versus non-Robotic Mitral Valve Surgery on Hospital Resource Utilization, Costs, and Reimbursements. AATS Mitral Conclave 2015. New York, April 23-24.

8. Shander A, Hofmann A, Ozawa S, et al. Activity-based costs of blood transfusions in surgical patients at four hospitals. Transfusion 2010;50:753-65.

9. Murphy DA, Moss E, Binongo J, et al. The Expanding Role of Endoscopic Robotics in Mitral Valve Surgery: 1,257 
Consecutive Procedures. Ann Thorac Surg 2015;100:167581; discussion 1681-2.

10. Grossi EA, Goldman S, Wolfe JA, et al. Minithoracotomy for mitral valve repair improves inpatient and postdischarge economic savings. J Thorac Cardiovasc Surg 2014;148:2818-22.e1-3.

11. Mihaljevic T, Koprivanac M, Kelava M, et al. Value of robotically assisted surgery for mitral valve disease. JAMA Surg 2014;149:679-86.

12. Suri RM, Antiel RM, Burkhart HM, et al. Quality of life after early mitral valve repair using conventional and robotic approaches. Ann Thorac Surg 2012;93:761-9.

13. Cheng DC, Martin J, Lal A, et al. Minimally invasive

Cite this article as: Moss E, Halkos ME. Cost effectiveness of robotic mitral valve surgery. Ann Cardiothorac Surg 2017;6(1):33-37. doi: 10.21037/acs.2017.01.03 versus conventional open mitral valve surgery: a metaanalysis and systematic review. Innovations (Phila) 2011;6:84-103.

14. LaPar DJ, Crosby IK, Rich JB, et al. A contemporary cost analysis of postoperative morbidity after coronary artery bypass grafting with and without concomitant aortic valve replacement to improve patient quality and cost-effective care. Ann Thorac Surg 2013;96:1621-7.

15. Ho C, Tsakonas E, Tran K, et al. Robot-Assisted Surgery Compared with Open Surgery and Laparoscopic Surgery: Clinical Effectiveness and Economic Analyses [Internet]. Ottawa (ON): Canadian Agency for Drugs and Technologies in Health; 2011. 